\title{
The Initial-Final Mass Relationship of White Dwarfs in Common Proper Motion Pairs and Open Clusters
}

\author{
S. Catalán ${ }^{1,2}$, I. Ribas ${ }^{1,2}$, J. Isern ${ }^{1,2}$, \\ E. García-Berro ${ }^{1,3}$ and C. Allende Prieto ${ }^{4}$ \\ ${ }^{1}$ Institut d'Estudis Espacials de Catalunya, c/ Gran Capità 2-4, 08034 Barcelona, Spain \\ email: catalan@ieec.uab.es \\ ${ }^{2}$ Institut de Ciències de l'Espai, CSIC, Facultat de Ciències, UAB, 08193 Bellaterra, Spain \\ ${ }^{3}$ Departament de Física Aplicada, Escola Politècnica Superior de Castelldefels, Universitat \\ Politècnica de Catalunya, Avda. del Canal Olímpic s/n, 08860 Castelldefels, Spain \\ ${ }^{4} \mathrm{McD}$ onald Observatory and Dept. of Astronomy, University of Texas, Austin, TX 78712, USA
}

\begin{abstract}
We have studied white dwarfs in common proper motion pairs (CPMPs) to improve the semi-empirical initial-final mass relationship of white dwarfs. In this contribution, we report new results obtained from spectroscopic observations of both members of several CPMPs composed of an F, G or K type star and a DA white dwarf.
\end{abstract}

Keywords. stars: white dwarfs, stars: abundances, stars: evolution, binaries: visual

\section{Introduction and observations}

The initial-final mass relationship connects the properties of a white dwarf with those of its main-sequence progenitor. This function is important for determining the ages of globular clusters and their distances, for studying the chemical evolution of galaxies, and also for understanding the properties of the galactic population of white dwarfs. Despite its relevance, this relationship is still poorly constrained. A promising approach to decrease the uncertainties is to study white dwarfs for which external constraints are available. This is the case of white dwarfs in common proper motion pairs (CPMPs). Important information of the white dwarf can be inferred from the study of the companion, since they were born at the same time and with the same initial chemical composition.

The list of CPMPs under study was selected from the available literature (Wegner \& Reid 1991, Silvestri et al. 2001). Each CPMP (11 in total) is composed of a white dwarf classified as DA and an F, G or K type star. The observations were carried out using a suite of telescope/instrument configurations. The white dwarf members were observed with the LCS spectrograph of the HJS (2.7-m) telescope at McDonald Observatory (Texas, USA) and with the TWIN spectrograph of the 3.5-m telescope at Calar Alto Observatory (CAHA, Almería, Spain), obtaining a FWHM resolving power of $\sim 4-5 \AA$. The FGK companions were observed with the FOCES echelle spectrograph of the 2.2-m telescope at CAHA and with the SARG echelle spectrograph at the TNG telescope in La Palma (Canary Islands, Spain) with resolutions of $R \sim 47000$ and $R \sim 57000$, respectively. These spectroscopic observations have revealed that only 5 of the 11 white dwarfs were in fact of the DA type, whereas the rest were misclassified. 
Table 1. Stellar parameters derived for the observed FGK stars.

\begin{tabular}{lcccc}
\hline Name & $T_{\text {eff }}(K)$ & $Z$ & $\log \left(L / L_{\odot}\right)$ & Age $(\mathrm{Gyr})$ \\
\hline $\mathrm{G} \mathrm{158-77^{1 }}$ & $4387 \pm 27$ & - & - & - \\
$\mathrm{BD}+441847$ & $5627 \pm 49$ & $0.006 \pm 0.003$ & $-0.188 \pm 0.059$ & $-{ }^{2}$ \\
$\mathrm{BD}+232539^{1}$ & $5666 \pm 48$ & - & - & - \\
$\mathrm{BD}+342473$ & $6268 \pm 68$ & $0.015 \pm 0.008$ & $0.369 \pm 0.109$ & $3.25 \pm 2.28$ \\
$\mathrm{BD}-085980$ & $5669 \pm 52$ & $0.007 \pm 0.004$ & $-0.151 \pm 0.040$ & $-{ }^{2}$ \\
\hline
\end{tabular}

${ }^{1}$ Not analyzed because of low $S / N$. More observations are underway.

${ }^{2}$ The ages obtained from isochrone fits are not reliable (see text).

Table 2. Stellar parameters derived for the observed white dwarfs.

\begin{tabular}{lrccccc}
\hline Name & \multicolumn{1}{c}{$T_{\text {eff }}(K)$} & $\log g$ & $M_{\mathrm{f}}\left(M_{\odot}\right)$ & $t_{\text {cool }}(\mathrm{Gyr})$ & $t_{\mathrm{ms}}(\mathrm{Gyr})$ & $M_{\mathrm{i}}\left(M_{\odot}\right)$ \\
\hline WD0023+109 & $10377 \pm 230$ & $7.92 \pm 0.08$ & $0.56 \pm 0.03$ & $0.49 \pm 0.08$ & - & - \\
WD0913+442 & $8918 \pm 111$ & $8.29 \pm 0.02$ & $0.78 \pm 0.01$ & $1.72 \pm 0.06$ & - & - \\
WD1304+227 & $10798 \pm 120$ & $8.21 \pm 0.05$ & $0.73 \pm 0.13$ & $0.73 \pm 0.06$ & - & - \\
WD1354+340 & $13650 \pm 437$ & $7.80 \pm 0.15$ & $0.49 \pm 0.10$ & $0.14 \pm 0.02$ & $3.11 \pm 2.28$ & $1.450_{-0.90}^{+0.215}$ \\
WD2253-081 & $7200 \pm 170$ & $8.40 \pm 0.08$ & $0.87 \pm 0.03$ & $3.75 \pm 0.19$ & - & - \\
\hline
\end{tabular}

\section{Analysis and results}

To determine the total age of each system, we analyzed the spectra of the FGK stars following this procedure: first, we derived the effective temperatures, $T_{\text {eff }}$, using the available $V J H K$ photometry and following the method of Masana et al. (2006). Then, we fitted the observed spectra using the SYNSPEC program (Hubeny \& Lanz 1995) and synthetic spectra based on Kurucz atmospheres to derive their metallicities, $Z$, focusing on spectral windows where unblended lines of FeI, FeII and NiI are present. If the distance and the apparent magnitude are known the calculation of the luminosity, $L$, is straightforward. Finally, we interpolated the stellar models of Schaller et al. 1992 using $T_{\text {eff }}, Z$ and $L$ to obtain the ages of these stars, and, consequently, the total ages of the CPMPs - see Table 1. We have found that some of the observed stars are fairly unevolved, so isochrone fits do not provide reliable ages. Other age indicators such as chromospheric activity or X-ray luminosity are currently being considered.

The atmospheric parameters of each white dwarf, $T_{\text {eff }}$ and $\log g$, were derived from the fitting of the theoretical models of D. Koester to the observed Balmer lines using the package SPECFIT of IRAF following the procedure described in Bergeron et al. (1992) - see Table 2 . Then, we derived its mass, $M_{\mathrm{f}}$, and cooling time, $t_{\text {cool }}$, using the cooling sequences of Salaris et al. (2000). Since we know the total age of the white dwarf (from the companion), we obtain the main sequence lifetime of the progenitor, $t_{\mathrm{ms}}$, by subtracting its cooling time to the age of the system. Finally, using the stellar models of Domínguez et al. (1999) we compute the mass of the progenitor in the main sequence, $M_{\mathrm{i}}$.

In Figure 1 we show the final versus initial mass of the white dwarf in a CPMP for which the analysis has been completed. Also plotted are the results for the white dwarfs in the open clusters M 35 (Williams et al. 2004) and M 37 (Kalirai et al. 2005). We used the $T_{\text {eff }}$ and $\log g$ reported by these authors and then, for internal consistency, we followed the same procedure as for the white dwarfs in our list. By comparing the observational data it can be noted that the error obtained for the $M_{\mathrm{i}}$ in the case of the white dwarf in a CPMP is higher than those obtained for white dwarfs in open clusters. In fact, the uncertainty in the determination of the ages is the main drawback of using CPMPs, but it can be minimized by selecting the pairs with evolved companions. 


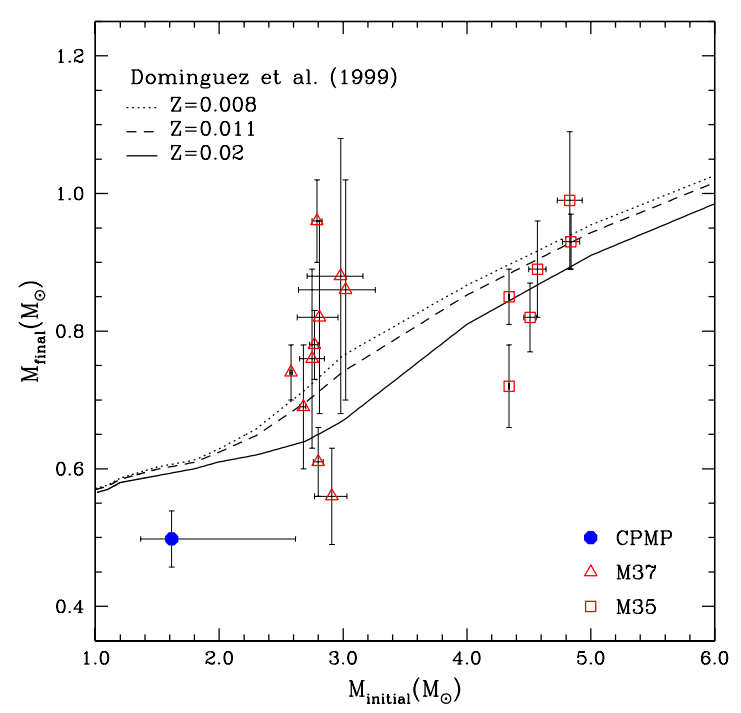

Figure 1. Final masses versus the initial masses obtained for one of the CPMPs in our sample (circle) and for white dwarfs in the open clusters M 35 (squares) and M 37 (triangles).

However, the fact that these systems are potentially more abundant and closer to us makes this a promising approach to improve the semi-empirical initial-final mass relation, since a better spectroscopic study of both members of the pairs can be done. White dwarfs in CPMPs also provide a wider age coverage of this relation, in contrast with open clusters, which are younger and do not contribute to its low-mass range.

A thorough comparison of the semi-empirical data with different theoretical relationships is currently underway and reveals a large scatter in the distribution (Figure 1). This could be due to the fact that maybe the initial-final mass relationship is not a single-valued function. More observational data and improved theoretical stellar models (mainly in the AGB phase) will help to better establish this function. In this sense, to further extend the sample, a cross-correlation of the SIMBAD database and Villanova White Dwarf Catalog has provided us with more pairs, which we expect to study shortly.

\section{Acknowledgements}

This research was partially supported by the MCYT grant AYA05-08013-C03-01 and 02, by the European Union FEDER funds and by the AGAUR.

\section{References}

Bergeron, P., Wesemael, F., \& Fontaine, G. 1992, ApJ, 387, 288

Domínguez, I., Chieffi, A., Limongi, M., \& Straniero, O. 1999, ApJ, 524, 226

Hubeny, I. \& Lanz, T. 1995, ApJ, 439, 875H

Kalirai, J.S., Richer, H.B., Reitzel, D., Hansen, B.M.S., Rich, R.M., Fahlman, G.G., Gibson, B.K., \& von Hippel, T. 2005, ApJ, 618, L123

Masana, E., Jordi, C., \& Ribas, I. 2006, A\& $A$, 450, 735

Salaris, M., García-Berro, E., Hernanz, M., Isern, J., \& Saumon, D. 2000, ApJ, 544, 1036

Schaller, G., Schaerer, D., Meynet, G., \& Maeder, A. 1992, A\&AS, 96, 269

Silvestri, N., Oswalt, T., Wood, M., Smith, J., Reid, I., \& Sion, E. 2001, AJ, 121, 503

Wegner, G. \& Reid, I.N. 1991, ApJ, 375, 674

Williams, K.A., Bolte, M., \& Koester, D. 2004, ApJ, 615, L49 